\title{
Wieviel Feldstärke ist notwendig für akzeptable portabel-indoor-Versorgung mit digitalem Rundfunk?
}

\author{
J. Philipp \\ Südwestrundfunk Stuttgart, Frequenz- und Versorgungsplanung, Germany
}

\begin{abstract}
Zusammenfassung. Durch eine Fragebogenaktion wurde ermittelt, welche Anforderungen potentielle Teilnehmer an die Güte des indoor-portabel-Empfangs von Radio und Fernsehen stellen. Mittels eines einfachen statistischen Feldstärkemodells wurde daraus abgeleitet, welche mittlere Mindestfeldstärke in $10 \mathrm{~m}$ Höhe über Grund bereit zu stellen ist, damit mindestens 95\% der im Erdgeschoss wohnenden Teilnehmer akzeptabel versorgt sind. Es ergibt sich für DAB im Band III ein um 6 bis $8 \mathrm{~dB}$ höherer Wert als der, welcher für mobile Versorgung mit $99 \%$ Ortswahrscheinlichkeit anzusetzen ist (und welcher den bisher geplanten Sendernetzen zu Grunde liegt). Für DVB-T im Band III sind mindestens $3 \mathrm{~dB}$ mehr anzusetzen als eine Rechnung nach "Chester"-Parametern bei 95\% Ortswahrscheinlichkeit verlangt, während bei DVB-T im Band V die Werte der 95\%Chester-Rechnung nur geringfügig nach oben korrigiert werden müssen. Die Ergebnisse für DVB-T gelten unter der Voraussetzung, dass ein $\mathrm{C} / \mathrm{N}$ von $17 \mathrm{~dB}$ für einen zufrieden stellenden Empfang ausreicht. Höhere Anforderungen an $\mathrm{C} / \mathrm{N}$ sind zusätzlich in Ansatz zu bringen.
\end{abstract}

\section{Einleitung}

Die Sendernetze für DigitalRadio (DAB) sind entsprechend den bei der CEPT-Planungskonferenz Wiesbaden 1995 (CEPT 1995) verwendeten Grundsätzen für Mobilempfang mit einer Ortswahrscheinlichkeit von 99\% geplant worden. Mittelfristig soll das System DAB das bisherige UKW-FM-Rundfunksystem ablösen. Eine der Vorbedingungen dafür ist jedoch, dass die Möglichkeit des portabelindoor-Empfangs besteht. Es muss also die Frage beantwortet werden, ob die bisher verwendeten Planungsparameter und Sendernetzkonfigurationen dieser Anforderung bereits genügen bzw. inwiefern Nachbesserungen erforderlich sind.

Correspondence to: J. Philipp (Johannes.Philipp@swr.de)
Eine verwandte Frage stellt sich beim digitalen terrestrischen Fernsehen (DVB-T). Für diesen Dienst werden Marktchancen nur gesehen, wenn bereits in der Startphase zumindest in Ballungsräumen portabel-indoor-Empfang möglich ist. Hier ist also insbesondere zu klären, ob die bei der Planungskonferenz "Chester 97" (CEPT 1997, EBU 1999) für die Empfangsart "portabel indoor" verwendeten Parameter ausreichen, um beim Teilnehmer und Kunden eine genügende Akzeptanz dieses Dienstes zu sichern.

Dazu wurde mittels einer Fragebogenaktion ermittelt, welche Zahl von Aufstellversuchen für das Empfangsgerät ein Teilnehmer zu tolerieren bereit ist (Akzeptanzmodell). Mittels eines statistischen Modells für die Verteilung der Feldstärke lässt sich daraus ein Zusammenhang zwischen der mittleren indoor-Feldstärke im Pixel und dem Anteil der frustrierten Teilnehmer ableiten. Das für die Planungstagung "Chester" verwendete Feldstärkemodell ist zur Kopplung mit dem Akzeptanzmodell allerdings nicht geeignet. Es ignoriert nämlich die räumlich kleinskaligen Feldstärkeschwankungen, obwohl genau diese dem Teilnehmer bei seinen Empfangsversuchen im Zimmer zu schaffen machen. Mit einem verfeinerten Feldstärkemodell lassen sich jedoch die zur definierten Begrenzung der Teilnehmerfrustration notwendigen Pegelüberschüsse quantifizieren. Diese ersetzen also die im Chester-Modell mit globalen Ortswahrscheinlichkeiten verknüpften Pegel-"Margins".

Die nachstehenden Überlegungen umschließen die Fälle der simultanen Empfangsmöglichkeit von bis zu 6 Multiplexen aus dem VHF- und UHF-Bereich sowohl in Empfangssituationen mit homogenem Außenpegel für alle betrachteten indoor-Situationen als auch in solchen Situationen, bei denen zwischen senderzu- und abgewandten Räumen unterschieden werden muss. 


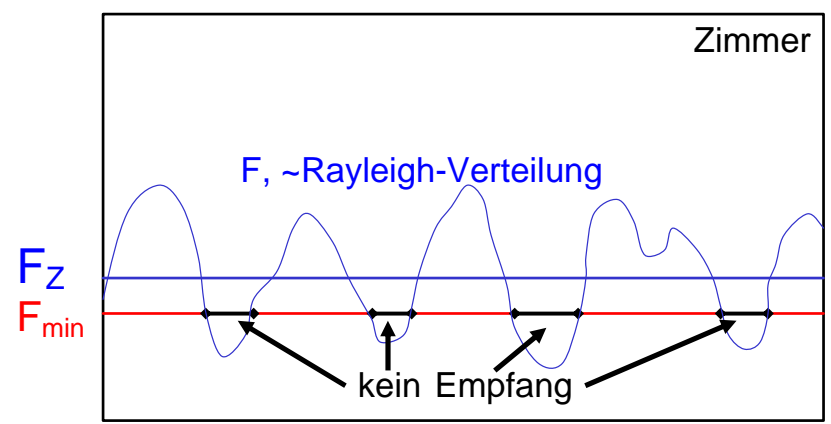

Abbildung 1. Empfangsverhältnisse in einem Zimmer. Wo der tatsächliche Pegel $F$ den Mindestpegel $F_{\text {min }}$ unterschreitet, ist kein Empfang möglich. Je mehr der mittlere Pegel $F_{Z}$ den Mindestpegel $F_{\text {min }}$ überschreitet, desto mehr Orte im Zimmer sind für das Aufstellen des Empfängers geeignet.

\section{Feldstärkemodell}

Der Feldstärkepegel in einem betrachteten Zimmer ist nicht konstant. Da das Wellenfeld die Überlagerung einer Primärwelle mit vielen durch Reflexion an den Wänden und der Einrichtung entstandenen Sekundärwellen darstellt, besitzt der Pegel Schwankungen, die näherungsweise durch eine Rayleigh-Verteilung beschrieben werden können. Nur solche Orte im Zimmer kommen für das Aufstellen eines Empfängers in Betracht, an denen der systemspezifische Mindestpegel $F_{\min }$ überschritten wird. Bild 1 illustriert die Empfangsverhältnisse in einem Zimmer.

Der Feldstärkepegel an einem festen Aufstellort für das Empfangsgerät in einem konkreten Zimmer ist gegeben durch den Mittelwert des Feldstärkepegels in diesem Zimmer und die als statistische Größe modellierte Abweichung des Pegels am Aufstellort von diesem Mittelwert:

$F=F_{Z}+\Delta F_{i}$

Dabei ist

$$
\begin{aligned}
& \mathrm{F}=\text { Feldstärkepegel am Aufstellort } \\
& \mathrm{F}_{Z}=\text { Mittelwert des Feldstärkepegels im Zimmer } \\
& \Delta \mathrm{F}_{i}=\text { Abweichung des Pegels am Aufstellort vom Mit- } \\
& \text { telwert im Zimmer. }
\end{aligned}
$$

Die Streuung des Feldstärkepegels im Zimmer wird für alle Zimmer als gleich angenommen und mit $\sigma_{i}$ bezeichnet.

Übliche Methoden der Feldstärkeberechnung ermöglichen es jedoch nicht, für jedes einzelne Zimmer einen Mittelpegel vorherzusagen. Die Feldstärkeprognose bezieht sich immer auf Planquadrate ("Pixel") mit linearen Abmessungen von einigen Hundert bis einigen Tausend Wellenlängen (z.B. $100 \mathrm{~m} \times 100 \mathrm{~m}$ ), welche jeweils eine Vielzahl von Zimmern umfassen und für die lediglich der Mittelwert des Feldstärkepegels in $10 \mathrm{~m}$ Höhe über Grund quasideterministisch angegeben werden kann.

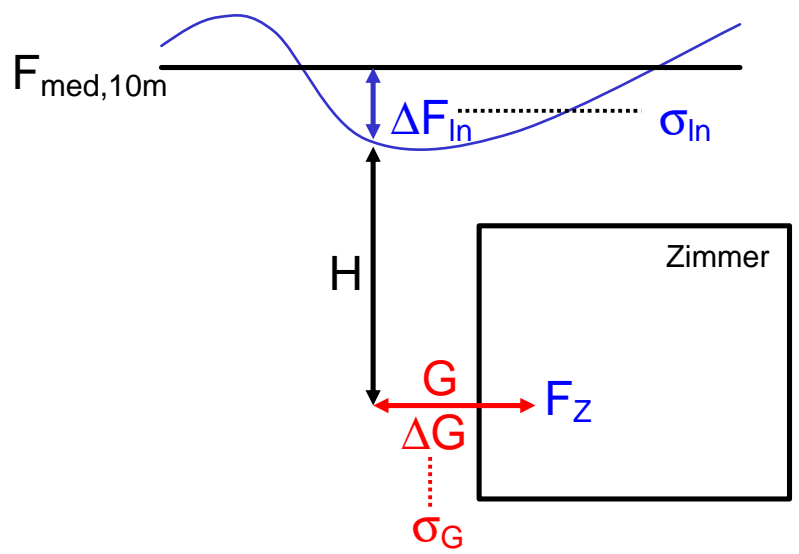

Abbildung 2. Das für die Betrachtungen verwendete Feldstärkemodell. Der mittlere Pegel im Zimmer, $F_{Z}$, hängt mit $\mathrm{F}_{\text {med,10m }}$, dem Pixel-Median in $10 \mathrm{~m}$ Höhe über Grund, über die aktuelle Pegelabweichung $\Delta F_{l n}$, die Höhenkorrektur $\mathrm{H}$ und die Gebäudedämpfung zusammen. Die Abweichung $\Delta F_{l n}$ des tatsächlichen Pegels in $10 \mathrm{~m}$ Höhe über Grund vor der Außenwand des Zimmers vom Median $F_{m e d}, 10 m$ besitzt die Streuung $\sigma_{l n}$. Die aktuelle Gebäudedämpfung setzt sich zusammen aus dem Mittelwert $G$ und die aktuelle Abweichung $\Delta G$, deren Streuung durch $\sigma_{G}$ gegeben ist.

Der Mittelwert des Feldstärkepegels in einem speziellen Zimmer im Erdgeschoss ergibt sich aus dem Mittelwert des Feldstärkepegels im Pixel in $10 \mathrm{~m}$ Höhe über Grund, abzüglich der Höhenkorrektur auf 1,5 m Höhe und abzüglich der Gebäudedämpfung:

$F_{Z}=F_{\text {med }, 10 \mathrm{~m}}+\Delta F_{l n}-H-\mathrm{G}+\Delta \mathrm{G}$

Dabei ist

$\mathrm{F}_{\text {med, } 10 \mathrm{~m}}=$ über das Pixel gemittelter Wert des Feldstärkepegels in $10 \mathrm{~m}$ Höhe über Grund

$\Delta \mathrm{F}_{l n}=$ normalverteilte Abweichung des Pegels in $10 \mathrm{~m}$ Höhe vor der Außenwand des Zimmers vom Mittelwert im Pixel

$\mathrm{H}=$ Feldstärkeabschlag für 1,5 m Höhe

$G=$ Mittelwert der Gebäudedämpfung

$\Delta \mathrm{G}=$ Abweichung der betrachteten Gebäudedämpfung vom Mittelwert.

Unter Gebäudedämpfung wird hier der Pegelunterschied zwischen dem räumlichen Mittelwert des Pegels vor der Außenwand (dem Fenster) des Zimmers und dem Mittelwert im Zimmer verstanden.

Der Feldstärkepegel in $10 \mathrm{~m}$ Höhe wird als normalverteilt angesehen; seine Streuung wird mit $\sigma_{l n}$ bezeichnet und bei den Beispielrechnungen mit 5,5 dB angesetzt.

Die Streuung der Gebäudedämpfung wird mit $\sigma_{G}$ bezeichnet. 
Bild 2 zeigt schematisch das Zustandekommen des Zimmer-Mittelpegels aus den verschiedenen Komponenten; die blaue Kurve symbolisiert den tatsächlichen Verlauf des Pegels in $10 \mathrm{~m}$ Höhe über Grund.

Entsprechend den bei der Chester-Planungstagung verwendeten Annahmen werden für die im Folgenden dargestellten Beispielrechnungen für VHF die Werte $G=8 \mathrm{~dB}$, $\sigma_{G}=3 \mathrm{~dB}$ und für UHF die Werte $G=7 \mathrm{~dB}, \sigma_{G}=6 \mathrm{~dB}$ verwendet.

Der Durchschnittswert der einzelnen Zimmer-Mittel des Feldstärkepegels über das Pixel wird mit $\overline{F_{Z}}$ bezeichnet und ist gegeben durch

$\overline{F_{Z}}=F_{\text {med }, 10 \mathrm{~m}}-H-G$

\section{Misserfolgswahrscheinlichkeit}

Zum Funktionieren des Empfängers ist der Mindestpegel $F_{\min }$ erforderlich. Die bedingte Wahrscheinlichkeit $p\left(F<F_{\min } \mid F_{Z}\right)$, mit welcher der tatsächliche Pegel $F$ am Aufstellort den Mindestpegel unterschreitet, wenn im betrachteten Zimmer der Mittelwert $F_{Z}$ vorliegt, ist gegeben durch

$p\left(F<F_{\min } \mid F_{Z}\right)=\Phi\left[\left(F_{\min }-F_{Z}\right) / \sigma_{i}\right]$

Dabei ist $\Phi(x)$ die von $-\infty$ bis $x$ kumulierte Wahrscheinlichkeit der normalisierten Normalverteilung.

Wird an einem vom Teilnehmer ausprobierten Aufstellort der Mindestpegel nicht erreicht, hat der Teilnehmer mit diesem Aufstellversuch einen Misserfolg. Die Wahrscheinlichkeit für einen Misserfolg bei einem Aufstellversuch in einem Zimmer mit mittlerem Pegel $F_{Z}$ ist natürlich gleich der Wahrscheinlichkeit $p\left(F<F_{\min } \mid F_{Z}\right)$. Die Wahrscheinlichkeit $p_{n}$, bei n Aufstellversuchen keinen Erfolg zu haben, ist dann entsprechend gegeben durch

$p_{n}=p^{n}$

Im betrachteten Pixel streuen die Zimmermittel $F_{Z}$ um das Mittel $\overline{F_{Z}}$. Über alle Zimmer des Pixels gesehen ist die Wahrscheinlichkeit $P_{1}\left(F<F_{\text {min }}\right)$, mit einem Aufstellversuch keinen Erfolg zu haben, gegeben durch den Mittelwert der bedingten Wahrscheinlichkeit $p_{1}\left(F<F_{\min } \mid F_{Z}\right)$ über alle vorkommenden Werte von $F_{Z}$ :

$P_{1}\left(F<F_{\min }\right)=\int_{-\infty}^{\infty} d F_{Z} \rho\left(F_{Z}\right) p_{1}\left(F<F_{\min } \mid F_{Z}\right)$

Dabei ist $\rho\left(F_{Z}\right)$ die Wahrscheinlichkeitsdichte des Vorliegens von $F_{Z}$ in einem Zimmer. Die als normal angenommene Verteilung der $F_{Z}$ hat den Mittelwert $\overline{F_{Z}}$ gemäß Gl. (3) und besitzt die Streuung $\sigma_{e}$, die gegeben ist durch

$\sigma_{e}=\sqrt{\sigma_{l n}^{2}+\sigma_{G}^{2}}$
Die über alle Zimmer des Pixels gesehene Wahrscheinlichkeit $P_{n}$, mit n Aufstellversuchen keinen Erfolg zu haben, ist entsprechend gegeben durch

$P_{n}=\int_{-\infty}^{\infty} d F_{Z} \rho\left(F_{Z}\right) p_{n}\left(F<F_{\min } \mid F_{Z}\right)$

was sich unter Berücksichtigung der Gl. (5) und (7) schreiben lässt als

$$
\begin{array}{r}
P_{n}=\frac{1}{\sqrt{2 \pi} \sigma_{e}} \int_{-\infty}^{\infty} d F_{Z} \exp \left[-\frac{\left(F_{Z}-\overline{F_{Z}}\right)^{2}}{2 \sigma_{e}^{2}}\right] \\
\Phi^{n}\left(\frac{F_{\min }-F_{Z}}{\sigma_{i}}\right)
\end{array}
$$

$P_{n}$ hängt nicht von $\overline{F_{Z}}$ und $F_{\min }$ separat ab, sondern nur von der Differenz

$D:=\overline{F_{Z}}-F_{\min }$

die im Folgenden als "Pegelüberschuss" bezeichnet werden soll.

Ersetzt man nämlich in Gl. (9) die Integrationsvariable $F_{Z}$ durch $x$, definiert als

$x:=F_{Z}-F_{\min }$

so vereinfacht sich Gl. (9) zu

$P_{n}=\frac{1}{\sqrt{2 \pi} \sigma_{e}} \int_{-\infty}^{\infty} d x \exp \left[-\frac{x^{2}}{2 \sigma_{e}^{2}}\right] \Phi^{n}\left(-\frac{x+D}{\sigma_{i}}\right)$

Für den Spezialfall $\sigma_{e}=\sigma_{i}$ und $D=0$ kann das Integral (12) elementar berechnet werden. Es ergibt sich dabei $P_{n}=1 /(n+1)$. Für $n=1$ ist der Wert des Integrals auch bei voneinander verschiedenen Streuungen $\sigma_{e}$ und $\sigma_{i}$ gleich $1 / 2$. Dies folgt aus der Tatsache, dass $\Phi\left(-x / \sigma_{i}\right)-1 / 2$ eine ungerade, der Exponentialausdruck im Integranden jedoch eine gerade Funktion ist.

Anmerkung: Das Komplement der Misserfolgswahrscheinlichkeit $P_{1}$, also die über alle betrachtete Zimmer gemittelte Wahrscheinlichkeit, mit welcher der tatsächliche Pegel $F$ den Mindestpegel $F_{\min }$ erreicht oder übersteigt, stellt die über die Zimmer gemittelte Ortswahrscheinlichkeit des Pegels im Zimmer dar. Diese ist streng zu unterscheiden von der bei Planungen mit dem Chester-Ansatz verwendeten Ortswahrscheinlichkeit! Diese entspricht nämlich der Wahrscheinlichkeit, mit welcher der durchschnittliche Mittelpegel $\overline{F_{Z}}$ den Mindestpegel $F_{\text {min }}$ erreicht oder überschreitet.

\section{Anteil der frustrierten Teilnehmer}

Sei $a_{n}$ der Anteil der Teilnehmer, der insgesamt $\mathrm{n}$ Aufstellversuche (also n-1 zusätzliche Aufstellversuche) toleriert. 


\section{SÜDWESTRUNDFUNK}

Frequenz- und Versorgungsplanung

70150 Stuttgart

Fragebogen zum ,indoor-portabel“-Empfang von

digitalem terrestrischen Fernsehen (DVB-T)

Bei DVB-T, dem digitalen terrestrischen Fernsehen, braucht man zum häuslichen Empfang keine Dachantenne und keine das Haus durchziehende Koaxialkabelverteilung. Die direkt am Empfangsgerät montierte Stabantenne reicht für einwandfreie Bild- und Tonqualität in Küche, Wohnund Schlafzimmer gewöhnlich aus.

Allerdings gibt es in jedem Raum für den Empfang günstigere und weniger günstige Stellen*. So kann es sein, dass der Empfänger z.B. gerade auf der Kommode, die sich zunächst als Aufstellort anbieten würde, nicht befriedigend funktioniert. Es ist dann notwendig, einen weiteren Aufstellort auszuprobieren. Manchmal genügt ein Verschieben des Gerätes um etwa eine Armlänge, manchmal muss eine ganz andere Ecke des Zimmers gewählt werden. Wieviele solcher weiteren Aufstellversuche halten Sie für zumutbar bzw. möglich (zutreffende Zahl bitte markieren)?

$\begin{array}{lllllllllll}0 & 1 & 2 & 3 & 4 & 5 & 6 & 7 & 8 & 9 & \geq 10\end{array}$

\section{Alter: \\ Geschlecht: männlich \\ weiblich.}

*: Die an den Zimmerwänden reflektierten Wellen überlagern sich zu einem komplizierten Wellenfeld, in dem sich Maxima und Minima der Feldstärke ausbilden. Befindet sich die Empfangsantenne zufällig in einem der Minima, kann es sein, dass der Pegel für einen einwandfreien Empfang nicht ausreicht.

Abbildung 3. Fragebogen zur Ermittlung des Akzeptanzverhaltens der Teilnehmer für indoor-portable-Empfang von Fernsehen. Der Fragebogen für DAB ist analog aufgebaut. Es fehlt lediglich der Hinweis auf "Bildqualität".

Der Anteil der Teilnehmer, der $\mathrm{n}$ Versuche toleriert, aber damit keinen Erfolg erzielt, ist dann gegeben durch das Produkt $a_{n} P_{n}$. Also ist bei gegebenem durchschnittlichen ZimmerMittelpegel $\overline{F_{Z}}$ der Anteil $U$ der Teilnehmer, der im Rahmen seiner individuellen Toleranz keinen Erfolg hat, gegeben durch

$U=\sum_{n=1}^{\infty} a_{n} P_{n}$

\section{Teilnehmerakzeptanz}

Die Fragebogen, mit denen das Akzeptanzverhalten der Teilnehmer ermittelt wurde, enthalten einen kurzen Einführungstext, mit welchem die Empfangssituation erläutert wird, eine Antwortzeile zum Markieren der Anzahl der tolerierten zusätzlichen Aufstellversuche sowie die Abfrage von Alter und Geschlecht. Als Beispiel ist der TV-Fragebogen in Bild 3 wiedergegeben.
Bei der Konzeption des Fragebogens wurde bewusst darauf verzichtet, die Probanden auf die Schwierigkeiten hinzuweisen, die sich ergeben können, wenn man versucht, an einem Aufstellort mehrere Multiplexe zu empfangen. Ebenso wurde darauf verzichtet, die Erleichterungen darzustellen, die eventuell mit abgesetzter Antenne möglich sind. Statt dessen wurde die Vorstellung eines Gerätes mit fest eingebauter Antenne vermittelt. Es wurde dem Teilnehmer überlassen, sich darunter ein stationäres "Wohnzimmer"-Gerät oder einen portablen Kleinempfänger vorzustellen.

Jede befragte Person wurde nur nach einem Dienst (entweder Radio oder Fernsehen) befragt, um den zu erwartenden Unterschied zwischen beiden Diensten nicht zu provozieren. Außerdem wurde darauf geachtet, dass nur solche Personen befragt wurden, die nicht über Fachkenntnisse der Hochfrequenztechnik verfügen, da zu vermuten ist, dass diese Personengruppe überdurchschnittlich viel Geduld bei den Aufstellversuchen an den Tag legt.

Zusätzlich zu der Zahl der akzeptierten Aufstellversuche wurde nach Alter und Geschlecht gefragt, um eventuelle Unausgewogenheiten der Probandenpopulation erkennen zu können. Auf den Aufwand der Erhebung der für eine wirklich repräsentative Umfrage notwendigen soziologischen Parameter musste allerdings verzichtet werden.

Die vorliegenden Auswertungen stützen sich auf einen Rücklauf von 128 Bögen für Fernsehen und 123 Bögen für Radio. Der jüngste befragte Teilnehmer war 15, der älteste 77 Jahre alt. Die Masse der Befragten verteilte sich auf die Altersgruppe der 20- bis 60-jährigen. Für beide Dienste wurden jeweils etwa gleich viele Männer und Frauen befragt.

Vereinzelt konnte beobachtet werden, dass Befragte sich nicht die Mühe machten, sich ihre individuelle Empfangssituation zu vergegenwärtigen, sondern dass sie die Skala der Antwortmöglichkeiten betrachteten $(0-10)$ und sich für den Mittelwert entschieden ("nehmen wir die goldene Mitte, also 5"). Im Ergebnishistogramm zeigte sich jedoch keine auffällige Häufung bei der Antwort " 5 ", so dass auf ein Ausfiltern der auf die beschriebene Weise zustande gekommenen Antworten verzichtet wurde.

Die beiden Grafiken dargestellt in den Bildern 4 und 5 zeigen die aus der Fragebogenaktion gewonnenen Anteile der Teilnehmer, die $n$ zusätzliche Versuche tolerieren, einmal für Radio und einmal für Fernsehen (im Sinne von Gl. (13) ist $a_{1}$ der Anteil derjenigen Teilnehmer, die 1 Versuch, also 0 zusätzliche Versuche tolerieren, etc.). Der Mittelwert der zusätzlich tolerierten Versuche liegt für Radio bei 3,3, für Fernsehen bei 2,5. Dieser Unterschied ist hochsignifikant $(p=0,013)$. Das Verhalten von Männern und Frauen unterscheidet sich bei beiden Diensten in gleicher Weise (Männer akzeptieren im Mittel geringfügig mehr Aufstellversuche). Allerdings ist dieser Unterschied statistisch nicht signifikant.

Instruktiv ist die Beantwortung der Frage, welche Misserfolgswahrscheinlichkeit in einem Zimmer höchstens gegeben sein darf, damit eine vorgegebene Frustrationsrate für Teilnehmer in diesem Zimmer nicht überschritten wird. Die 


\section{Radio: rel. Häufigkeit}

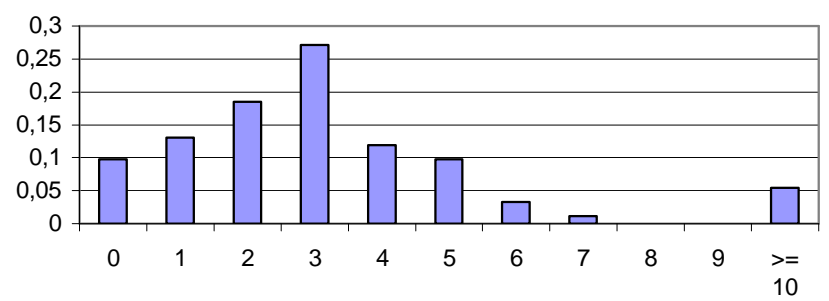

Anzahl tolerierter zusätzlicher Versuche

Abbildung 4. Histogramm der tolerierten zusätzlichen Aufstellversuche für das portable Empfangsgerät beim Dienst Radio. Der Mittelwert ist 3,3.

Antwort lässt sich gewinnen, indem man den Fall betrachtet, dass alle Zimmer den gleichen Mittelpegel $F_{Z}$ besitzen. Dann wird aus Gl. (13)

$U=\sum_{n=1}^{\infty} a_{n} p^{n}$

Setzt man $U=5 \%$ und löst man Gl. (14) mit den aus den Umfrageergebnissen stammenden Fernseh- bzw. RadioKoeffizienten nach $p$ auf, so erhält man für Fernsehen $p=20 \%$ und für Radio $p=31 \%$. Damit ergibt sich die notwendige Zimmer-Ortswahrscheinlichkeit für Fernsehen zu $80 \%$ und für Radio zu $69 \%$.

\section{Empfang mehrerer Multiplexe}

Es ist davon auszugehen, dass Teilnehmer mit dem "indoor" aufgestellten Empfangsgerät mehrere Multiplexe empfangen möchten, ohne jeweils die Antenne verrücken zu müssen. Die angestellten Betrachtungen sind also für diesen Fall zu verallgemeinern.

Dazu sei vereinfachend angenommen, dass alle Multiplexe aus demselben Frequenzbereich (VHF bzw. UHF) stammen und mit gleicher Leistung von den gleichen Standorten abgestrahlt werden. Dann darf angenommen werden, dass in jedem beliebigen der betrachteten Zimmer der PegelMittelwert für alle Multiplexe derselbe ist. $F_{Z}$ soll also zwar vom betrachteten Zimmer, jedoch nicht vom betrachteten Multiplex abhängen. Unter dieser Voraussetzung ist die Wahrscheinlichkeit, bei vorgegebenem $F_{Z}$ den i-ten Multiplex nicht empfangen zu können, gleich dem durch Gl. (6) gegeben Ausdruck $p$. Entsprechend ist - unkorrelierte Verteilungen der Einzelpegel im Zimmer vorausgesetzt - die (hinsichtlich $F_{Z}$ bedingte) Wahrscheinlichkeit, alle $m$ betrachteten Multiplexe an einem vorgegebenen Aufstellort empfangen zu können, gegeben durch $(1-p)^{m}$. Damit ergibt sich die Misserfolgswahrscheinlichkeit $p(m)$, also die
TV: rel. Häufigkeit

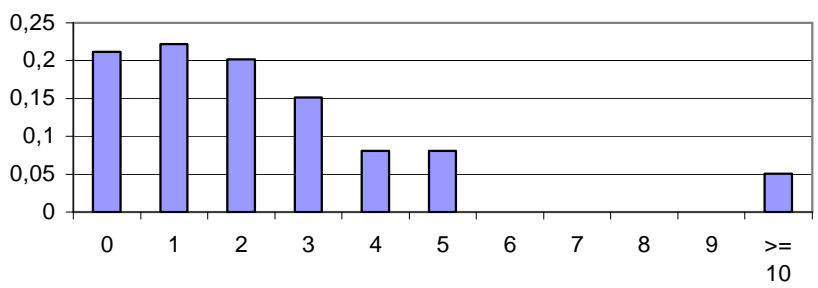

Anzahl tolerierter zusätzlicher Versuche

Abbildung 5. Histogramm der tolerierten zusätzlichen Aufstellversuche für das portable Empfangsgerät beim Dienst Fernsehen. Der Mittelwert ist 2,5.

Wahrscheinlichkeit, an einem gegebenen Aufstellort nicht alle $m$ Multiplexe empfangen zu können, zu

$p(m)=1-(1-p)^{m}$

Entsprechend Gl. (7) ist die bedingte Wahrscheinlichkeit $p_{n}(m)$, bei n Aufstellversuchen nicht alle $m$ Multiplexe empfangen zu können, gegeben durch

$p_{n}(m)=\left[1-(1-p)^{m}\right]^{n}$

Der Mittelwert von Gl. (16) über alle im Pixel vorkommenden $F_{Z}$ ist dann die Wahrscheinlichkeit $P_{n}(m)$, dass bei $\mathrm{n}$ Aufstellversuchen nicht alle $m$ Multiplexe empfangen werden können. Er ist entsprechend zu Gl. (12) gegeben durch

$$
\begin{aligned}
P_{n}(m)=\frac{1}{\sqrt{2 \pi} \sigma_{e}} & \int_{-\infty}^{\infty} d x \exp \left[-\frac{x^{2}}{2 \sigma_{e}^{2}}\right] \\
& \left\{1-\left[1-\Phi\left(-\frac{x+D}{\sigma_{i}}\right)\right]^{m}\right\}^{n}
\end{aligned}
$$

Für $m=1$ reduziert sich die Formel auf den Ausdruck (12). Für $\sigma_{e}=\sigma_{i}$ und $D=0$ ist das Integral elementar auswertbar. Es hat dann den Wert fac ${ }_{m}(n m) / \mathrm{fac}_{m}(n m+1)$, wobei fac ${ }_{m}()$ die $m$-fache Multifakultät bedeutet. Damit sind speziell

$\operatorname{fac}_{m}(n m)=m^{*} 2 m^{*} 3 m \ldots{ }^{*} n m$

und

$\operatorname{fac}_{m}(m n+1)=(m+1)^{*}(2 m+1) \ldots{ }^{*}(n m+1)$.

\section{Berücksichtigung senderzu- und abgewandter Zimmer}

Insbesondere am Rande des Versorgungsgebietes kann der Fall eintreten, dass senderabgewandte Zimmer mit deutlich niedrigerem Pegel versorgt werden als senderzugewandte. Der für abgewandte Zimmer in Anschlag zu bringende Pegelunterschied sei mit $A$ bezeichnet und es sei angenommen, 
1 Multiplex VHF

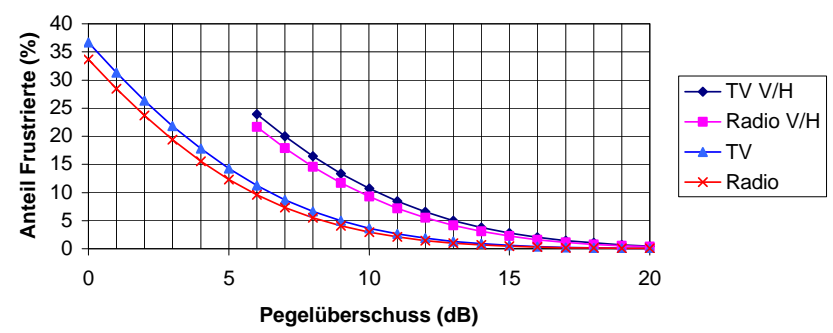

Abbildung 6. Frustrationswahrscheinlichkeit als Funktion des Pegelüberschusses bei Empfang von einem Multiplex (VHF). Die beiden unteren Kurven gehen von einer homogenen Pegelsituation für alle Zimmer aus. Die beiden oberen Kurven (TV V/H u. Radio V/H) berücksichtigen die Annahme, dass senderabgewandte Zimmer mit einem um $6 \mathrm{~dB}$ geringeren Pegel versorgt werden. Zum Erreichen der gleichen Frustrationswahrscheinlichkeit wie im homogenen Fall erfordert dies einen um ca. $4 \mathrm{~dB}$ erhöhten Pegelüberschuss der zugewandten Zimmer.

2 Multiplexe VHF

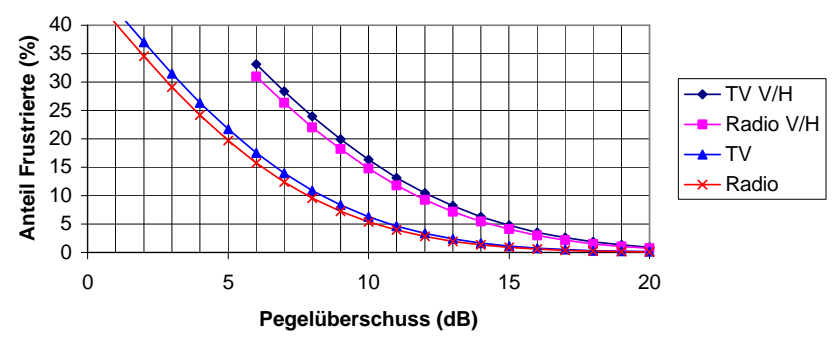

Abbildung 7. Frustrationswahrscheinlichkeit als Funktion des Pegelüberschusses bei Empfang von zwei Multiplexen (VHF). [V/HKurven: Senderabgewandte Zimmer werden mit einem $6 \mathrm{~dB}$ geringeren Pegel versorgt.] Bei zwei Multiplexen ist zum Erreichen der gleichen Frustrationswahrscheinlichkeit wie beim Empfang eines Multiplexes ein um ca. 2 dB höherer Pegelüberschuss erforderlich.

dass es gleich viele zu- wie abgewandte Zimmer gibt. Es sei $D$ der Pegelüberschuss der zugewandten Zimmer. Die entsprechenden Misserfolgswahrscheinlichkeiten $P_{(z u) n}(m)$ sind durch die $P_{n}(m)$ aus Gl. (17) gegeben:

$P_{(z u) n}(m)[D]=P_{n}(m)[D]$

Für die abgewandten Zimmer beträgt der Pegelüberschuss nur $D-A$. Daher ergeben sich die auf den Pegelüberschuss $D$ der zugewandten Zimmer bezogenen Misserfolgswahrscheinlichkeiten $P_{(a b) n}(m)$, indem auf der rechten Seite von Gl. (18) $D$ durch $D-A$ ersetzt wird:

$P_{(a b) n}(m)[D]=P_{n}(m)[D-A]$
6 Multiplexe VHF

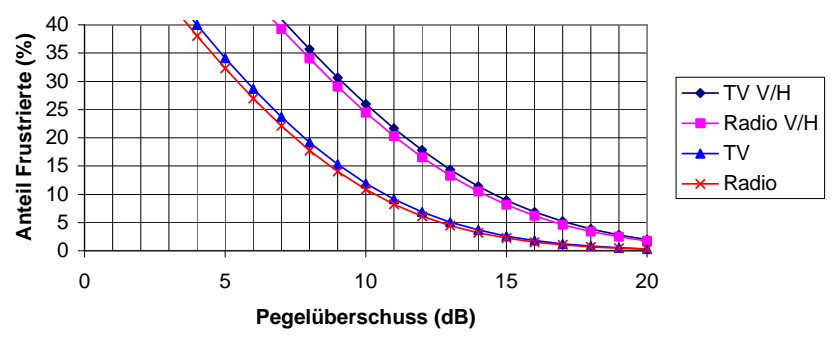

Abbildung 8. Frustrationswahrscheinlichkeit als Funktion des Pegelüberschusses bei Empfang von 6 Multiplexen (VHF). [V/HKurven: Senderabgewandte Zimmer werden mit einem $6 \mathrm{~dB}$ geringeren Pegel versorgt.] Bei 6 Multiplexen ist zum Erreichen der gleichen Frustrationswahrscheinlichkeit wie beim Empfang eines Multiplexes ein um ca. 4 dB höherer Pegelüberschuss erforderlich.

Der Anteil der unzufriedenen Teilnehmer ist analog zu Gl. (13) dann gegeben durch

$$
\begin{aligned}
U_{z u, a b} & =1 / 2 \sum_{i=1}^{\infty} a_{i}\left\{P_{i}(m)[D]+P_{i}(m)[D-A]\right\} \\
& =1 / 2(U[D]+U[D-A])
\end{aligned}
$$

\section{Frustrationswahrscheinlichkeit als Funktion des Pegelüberschusses}

Mit den aus der Fragebogenaktion ermittelten Koeffizienten $a_{i}$ lässt sich durch numerische Integration von Gl. (17) und Anwendung von Gl. (14) bzw. Gl. (20) die Frustrationswahrscheinlichkeit als Funktion des Pegelüberschusses $D$ (definiert in Gl. (10) berechnen. Die Ergebnisse für beide Dienste sind in den Grafiken (Bilder 6-13) wiedergegeben. Dabei wurden die Annahmen $\sigma_{l n}=5,5 \mathrm{~dB}, \sigma_{G}=3 \mathrm{~dB}$ (VHF) bzw. $\sigma_{G}=6 \mathrm{~dB}(\mathrm{UHF})$ und $\sigma_{i}=3,0 \mathrm{~dB}$ verwendet.

\section{Folgerungen}

\subsection{Radio}

Für DAB im Band III ist eine Mindestfeldstärke von $35 \mathrm{~dB}(\mu \mathrm{V} / \mathrm{m})$ erforderlich. Der erforderliche Pegelüberschuss für den Empfang von 3 Multiplexen (ohne Berücksichtigung eventuell schlechter versorgter senderabgewandter Zimmer) bei höchstens 5\% Frustration beträgt ca. $11 \mathrm{~dB}$ (6 Multiplexe erfordern $13 \mathrm{~dB}$ ). Zuzüglich einer mittleren Gebäudedämpfung von $8 \mathrm{~dB}$ und der Höhenkorrektur von weiteren $10 \mathrm{~dB}$ ergibt sich ein erforderlicher Mittelpegel von $64 \mathrm{~dB}(\mu \mathrm{V} / \mathrm{m})$ in $10 \mathrm{~m}$ Höhe über Grund. Dies sind $6 \mathrm{~dB}$ mehr ( $8 \mathrm{~dB}$ bei 6 Multiplexen) als die für Mobilversorgung mit $99 \%$ Ortswahrscheinlichkeit erforderlichen $58 \mathrm{~dB}(\mu \mathrm{V} / \mathrm{m})$. 
notw. Pegelüberschuss für m Multiplexe (VHF)

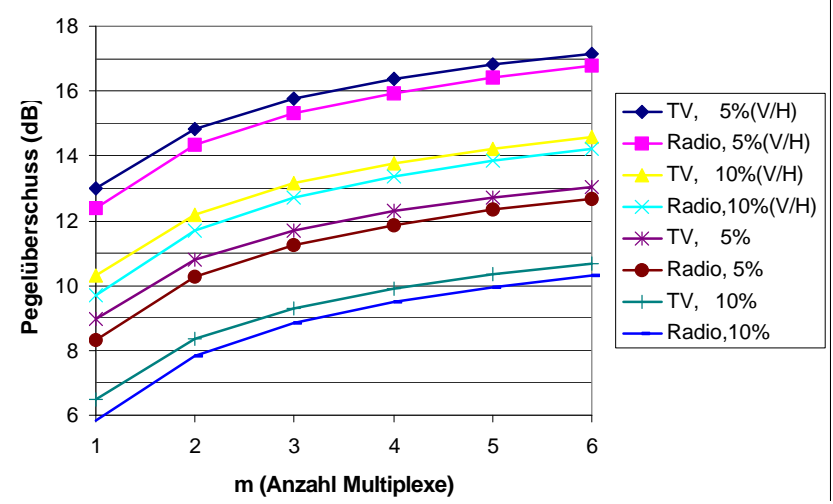

Abbildung 9. Notwendiger Pegelüberschuss in Abhängigkeit von der gewünschten Anzahl von Multiplexen (VHF) für Frustrationswahrscheinlichkeiten von $5 \%$ und $10 \%$

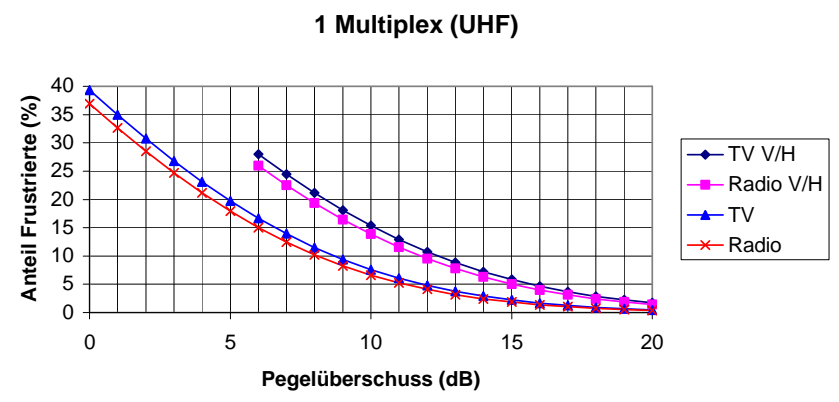

Abbildung 10. Frustrationswahrscheinlichkeit als Funktion des Pegelüberschusses bei Empfang von einem Multiplex (UHF). Die beiden unteren Kurven gehen von einer homogenen Pegelsituation für alle Zimmer aus. Die beiden oberen Kurven (TV V/H und Radio V/H) berücksichtigen die Annahme, dass senderabgewandte Zimmer mit einem um $6 \mathrm{~dB}$ geringeren Pegel versorgt werden. Zum Erreichen der gleichen Frustrationswahrscheinlichkeit wie im homogenen Fall erfordert dies einen um ca. 4 dB erhöhten Pegelüberschuss der zugewandten Zimmer.

Bei einem Mittelpegel von $58 \mathrm{~dB}(\mu \mathrm{V} / \mathrm{m})$ beträgt der Pegelüberschuss noch $5 \mathrm{~dB}$. Die zugehörige Frustrationswahrscheinlichkeit für den Empfang von nur 2 Multiplexen beträgt bereits $20 \%$. Am Rande des Versorgungsgebietes könnte dies eventuell noch tolerabel erscheinen. Allerdings kann es notwendig werden, gerade dort den Effekt senderzu- und abgewandter Zimmer zu berücksichtigen. Zur genaueren Beurteilung sind Untersuchungen realistischer Sendernetzkonfigurationen erforderlich.

Zum Vergleich kann die Rechnung auch für UKW-FM angestellt werden. Hierbei sind für Mono-Empfang mit einer $-3 \mathrm{~dB}$-Antenne in $10 \mathrm{~m}$ Höhe über Grund $48 \mathrm{~dB}(\mu \mathrm{V} / \mathrm{m})$ erforderlich. Unter Berücksichtigung einer Kabeldämpfung von $3 \mathrm{~dB}$ bedeutet dies für einen portablen Mono-Empfänger mit eingebauter -3dB-Antenne eine Mindestfeldstärke von $45 \mathrm{~dB}(\mu \mathrm{V} / \mathrm{m})$. Zur Ermittlung des notwendigen Pegelüber-
2 Multiplexe (UHF)

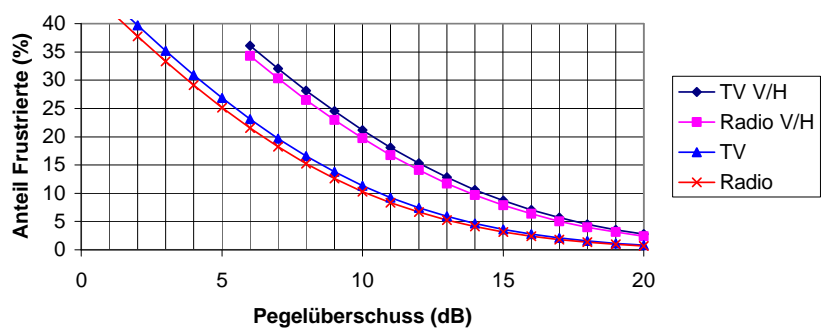

Abbildung 11. Frustrationswahrscheinlichkeit als Funktion des Pegelüberschusses bei Empfang von zwei Multiplexen (UHF). [V/HKurven: Senderabgewandte Zimmer werden mit einem $6 \mathrm{~dB}$ geringeren Pegel versorgt.]

6 Multiplexe UHF

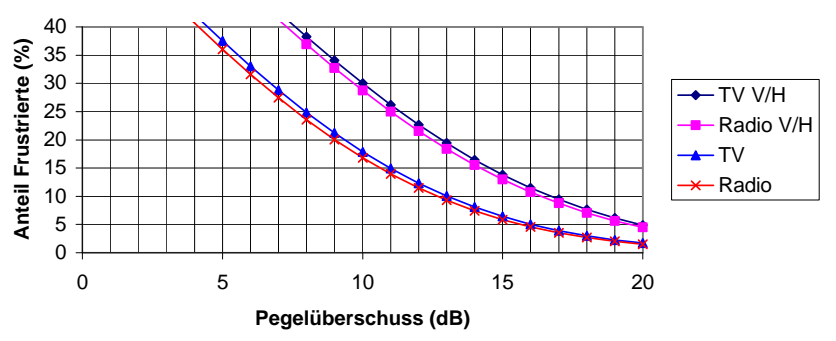

Abbildung 12. Frustrationswahrscheinlichkeit als Funktion des Pegelüberschusses bei Empfang von 6 Multiplexen (UHF). [V/HKurven: Senderabgewandte Zimmer werden mit einem $6 \mathrm{~dB}$ geringeren Pegel versorgt.] Bei 6 Multiplexen ist zum Erreichen der gleichen Frustrationswahrscheinlichkeit wie beim Empfang eines Multiplexes ein um ca. $5 \mathrm{~dB}$ höherer Pegelüberschuss erforderlich.

schusses ist hierbei von $\sigma_{l n}=8,3 \mathrm{~dB}$ auszugehen. Die Rechnung ergibt für den Empfang eines Programms einen Wert von $15 \mathrm{~dB}$. Zuzüglich der Gebäudedämpfung von $8 \mathrm{~dB}$ und dem Höhenabschlag von $10 \mathrm{~dB}$ ergibt sich daraus ein notwendiger mittlerer Feldstärkepegel in $10 \mathrm{~m}$ Höhe über Grund von $78 \mathrm{~dB}(\mu \mathrm{V} / \mathrm{m})$ für akzeptablen indoor-Empfang von UKW im Erdgeschoss.

Bei FM-Stereo kann von der nach der Versorgungsrichtlinie erforderlichen Mindestfeldstärke von $54 \mathrm{~dB}(\mu \mathrm{V} / \mathrm{m})$ in $10 \mathrm{~m}$ Höhe über Grund ausgegangen werden, die für die Verwendung einer Antenne mit $6 \mathrm{~dB}$ Gewinn gilt. Unter Berücksichtigung der wegfallenden Kabeldämpfung von $3 \mathrm{~dB}$ wären für einen portablen Empfänger mit $-3 \mathrm{~dB}$-Antenne dann $60 \mathrm{~dB}(\mu \mathrm{V} / \mathrm{m})$ für einwandfreien Stereo-Empfang erforderlich. Zuzüglich Pegelüberschuss für 1 Programm, Gebäudedämpfung und Höhenabschlag (Werte wie bei FM/Mono) ergibt sich ein notwendiger mittlerer Feldstärkepegel von $93 \mathrm{~dB}(\mu \mathrm{V} / \mathrm{m})$ in $10 \mathrm{~m}$ Höhe über Grund. 
notw. Pegelüberschuss für m Multiplexe (UHF)

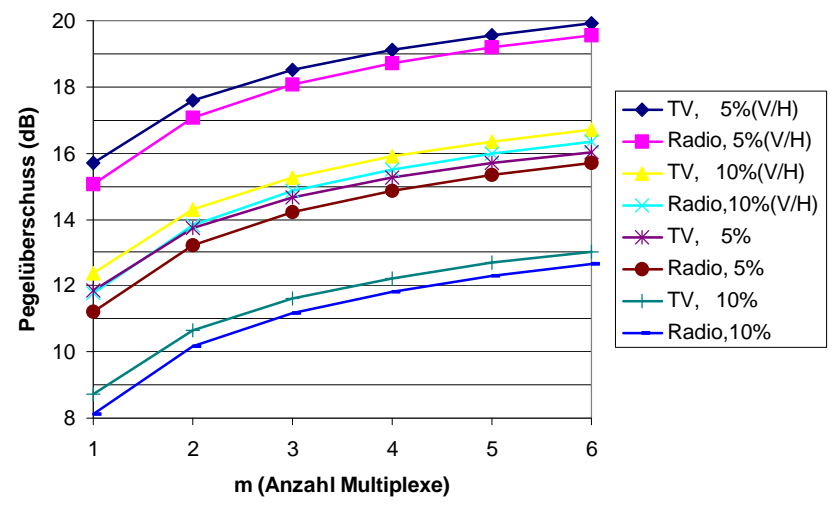

Abbildung 13. Notwendiger Pegelüberschuss bei Empfang von 1 bis 6 Multiplexen (UHF) für Frustrationswahrscheinlichkeiten von $5 \%$ und $10 \%$.

\subsection{Fernsehen}

Für DVB-T im Band III ist eine Mindestfeldstärke von $43 \mathrm{~dB}(\mu \mathrm{V} / \mathrm{m})$ erforderlich. Zuzüglich dem notwendigen Pegelüberschuss von $9 \mathrm{~dB}$ für einen Multiplex, der mittleren Gebäudedämpfung von $8 \mathrm{~dB}$ und der Höhenkorrektur von $10 \mathrm{~dB}$ ergibt sich ein erforderlicher Mittelpegel von $70 \mathrm{~dB}(\mu \mathrm{V} / \mathrm{m})$ in $10 \mathrm{~m}$ Höhe über Grund. Dies entspricht nahezu dem nach herkömmlicher Rechnung für 95\% Ortswahrscheinlichkeit anzusetzenden Wert von $71 \mathrm{~dB}(\mu \mathrm{V} / \mathrm{m})$. Allerdings ist davon auszugehen, dass der Empfang von mehr als einem Multiplex gewünscht wird. Da in VHF voraussichtlich nur ein Multiplex empfangbar sein wird, wäre es notwendig, die Statistik für den gemischten Fall "1 VHF-Multiplex + 5 UHF-Multiplexe" auszuarbeiten. In erster Näherung kann jedoch davon ausgegangen werden, dass es genügt, sowohl für den VHF-Multiplex als auch für die UHF-Multiplexe die Feldstärke zu verlangen, die notwendig ist, um simultan jeweils 6 VHF- bzw. UHF-Multiplexe verfügbar zu machen (da es darauf ankommt, im Zimmer jeweils die entsprechende Ortswahrscheinlichkeit zu erzeugen). Damit steigt der erforderliche Pegelüberschuss in VHF auf $13 \mathrm{~dB}$, was zu einer erforderlichen Mindestfeldstärke von $74 \mathrm{~dB}(\mu \mathrm{V} / \mathrm{m})$ führt (3 dB mehr als nach "Chester-95\%"-Rechnung).

Der für die "Chester"-Ortswahrscheinlichkeit von $95 \%$ im Band V erforderliche Mittelpegel von $85 \mathrm{~dB}(\mu \mathrm{V} / \mathrm{m})$ entspricht einem Pegelüberschuss von $14 \mathrm{~dB}$. Dies ist ausreichend für den Empfang von 2 Multiplexen auf einem Frustrationsniveau von 5\%. Beim Empfang von 6 Multiplexen entspricht der Pegelüberschuss von $14 \mathrm{~dB}$ einem Frustrationsniveau von etwa $8 \%$. Um das Frustrationsniveau auf 5\% zu begrenzen, wären zusätzlich $2 \mathrm{~dB}$ mehr Pegel erforderlich.

Obige Beispiele gelten nur unter den im Abschnitt 7 gemachten Annahmen für die Streuungen der zu betrachtenden statistischen Variablen. Ebenso wurde von einem erforderlichen $\mathrm{C} / \mathrm{N}$ von $14 \mathrm{~dB}$ für den Rayleigh-Kanal bei DVB-T ausgegangen sowie einer Implementationsmarge von $3 \mathrm{~dB}$. Es gibt jedoch erste aus Kanalsimulationen des Instituts für Rundfunktechnik stammende Hinweise, dass die tatsächlich vorhandene Zeitvarianz des portabel-indoor-Kanals einen weiteren Zuschlag für das notwendige $\mathrm{C} / \mathrm{N}$ erfordert. Außerdem ist bei konkreten Sendernetzplanungen zu berücksichtigen, dass der Höhenabschlag von $10 \mathrm{~dB}$ bzw. $12 \mathrm{~dB}$ bei manchen Bebauungskonfigurationen (z.B. hohe Wohnblocks mit Innenhöfen) zu niedrig angesetzt sein kann.

\section{Literatur}

CEPT 1995: Schlussakten der T-DAB-Planungstagung der CEPT Wiesbaden, 1995

CEPT 1997: The Chester 1997 Multilateral Coordination Agreement relating to Technical Criteria, Coordination Principles and Procedures for the introduction of Terrestrial Video Broadcasting (DVB-T), 1997

EBU 1999: ERC/EBU-Report on Planning and Introduction of Terrestrial Digital Television (DVB-T) in Europe, BPN 018, Izmir, 1999 